\title{
Design and Operation of Egg Collectors for Pelagic Spawning Marine Fishes ${ }^{1}$
}

\author{
Cortney Ohs, John Marcellus, Eric Cassiano, and Jason Broach ${ }^{2}$
}

\section{Introduction}

As aquaculture production techniques streamline, interest in refining captive breeding in marine fish species increases. The bait, food, and ornamental fish aquaculture industries all benefit from development of aquaculture techniques for desirable marine fish species. A few of the significant limitations on the development of aquaculture protocols for marine fish species are broodstock management, viable egg production, and larval development. A critical step in aquaculture production is to collect fertilized embryos, usually referred to as eggs, for examination and further development. Efficient egg collection is key to the success of an aquaculture operation.

Although marine fish use many different egg production strategies, many fall into two categories: demersal and pelagic. Demersal spawning fish usually deposit sticky eggs onto substrate near the tank bottom. Demersal egg production starts by initiating fish to spawn on removable substrate such as tile or PVC pipe. The substrate with attached eggs can then be moved into a separate tank for hatching.

Pelagic spawning fish simultaneously release sperm and eggs into the water where fertilization and embryo development take place. Once fertilized, pelagic eggs float and are at the mercy of the water current. In a tank, pelagic eggs must be removed speedily, before they can enter the recirculating aquaculture system components, where they would be destroyed. A properly designed egg collector and egg collection techniques must therefore be implemented to ensure timely and efficient collection and prevent pelagic eggs from being damaged. This publication includes directions for construction and operation of two types of pelagic egg collectors: pre-filter and floating airlift.

\section{Tanks for Egg Collection}

For pelagic spawning marine fish species, floating eggs are more likely to be viable than sinking eggs. However, examination of sinking eggs can help increase production efficiency, so collecting and evaluating all eggs spawned can be informative. There are numerous variables that can contribute to the percentage of sinking eggs, such as water quality, broodstock dynamics, and breeding environment. Therefore, having a good understanding of your target species and your recirculating system dynamics will improve production.

Many types of tanks are used to hold marine fish broodstock. Cylindrical tanks are usually already engineered to create a circular flow of water for cleaning purposes and fish movement. Circular flow is also ideal for manipulating

1. This document is FA211, one of a series of the Program in Fisheries and Aquatic Sciences of the School of Forest Resources and Conservation, UF/IFAS Extension. Original publication date February2019. Visit the EDIS website at https://edis.ifas.ufl.edu for the currently supported version of this publication.

2. Cortney Ohs, associate professor, Program in Fisheries and Aquatic Sciences of the School of Forest Resources and Conservation, Indian River Research and Education Center; John Marcellus, technician, Program in Fisheries and Aquatic Sciences, School of Forest Resources and Conservation, UF/IFAS Indian River Research and Education Center; Eric Cassiano, assistant Extension scientist, Program in Fisheries and Aquatic Sciences, School of Forest Resources and Conservation, Tropical Aquaculture Laboratory; and Jason Broach, post-doctoral assistant, Program in Fisheries and Aquatic Sciences of the School of Forest Resources and Conservation, Indian River Research and Education Center; UF/IFAS Extension, Gainesville, FL 32611.

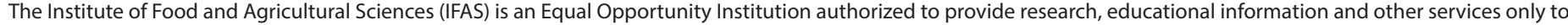

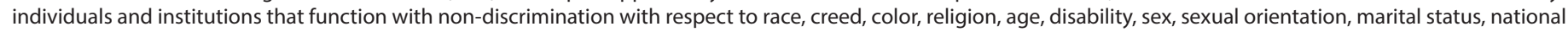
origin, political opinions or affiliations. For more information on obtaining other UF/IFAS Extension publications, contact your county's UF/IFAS Extension office. 
floating egg direction and collection. Rectangular tanks can be used to collect floating eggs as well, but surface water must be directed towards the egg collection device in these tanks, or the eggs can become stranded in the tank corners. Air flow from one side of the tank can direct the water toward the egg collector if strategically positioned. When collecting eggs, you should consider the safety of your broodstock. Depending on the size and shape of your tank as well as the behavior of the target species, you may have to take steps to keep your broodstock out of the egg collectors. Usually, a net or screen over the collection pipe is sufficient. The holes in the screen must be large enough to allow eggs to pass through, but small enough to keep your broodstock contained. Techniques will vary depending on species.

Two types of egg collectors will be described in this publication: pre-filter egg collectors and floating airlift egg collectors. Pre-filter egg collectors (Figures 1 and 2) are built into the overall recirculating aquaculture system and can be designed to provide the proper flow rate to avoid damaging eggs. Floating airlift egg collectors (Figure 3) are deployed inside an existing recirculating aquaculture system and are used in a variety of tanks and sumps, from small home tanks to large exhibits at public aquaria. Tank design and water flow rate will dictate the appropriate egg collection approach.

\section{Pre-Filter Egg Collector}

Pre-filter egg collectors are usually built as a part of the recirculating aquaculture system. In the water flow pathway of the system, they are implemented between the tank and the filtration, hence the name "pre-filter." If desired, the recirculating system can be constructed so that the flow of water bypasses the egg collector when needed. There are two general pre-filter egg collector designs: overflowing and upwelling.

A common overflow egg collector used in marine fish aquaculture is pictured in Figure 1. In this design, water and buoyant eggs enter through a skimmer pipe in the culture tank and flow directly into the egg collector, which is housed inside of a collection sump. Often the skimmer pipe will have an attached PVC pipe cut in half lengthwise to form a barrier for eggs as they rotate within the circular flow of the tank's surface water. This half pipe will direct floating eggs into the skimmer pipe, which exits the tank and flows directly into the egg collector. Another option for an overflow pre-filter egg collector is a modified plankton tow net (Figure 4). This maximizes surface area of the egg collector to prevent clogging of the screen, and will accommodate higher water flow rates and higher volumes of eggs spawned.

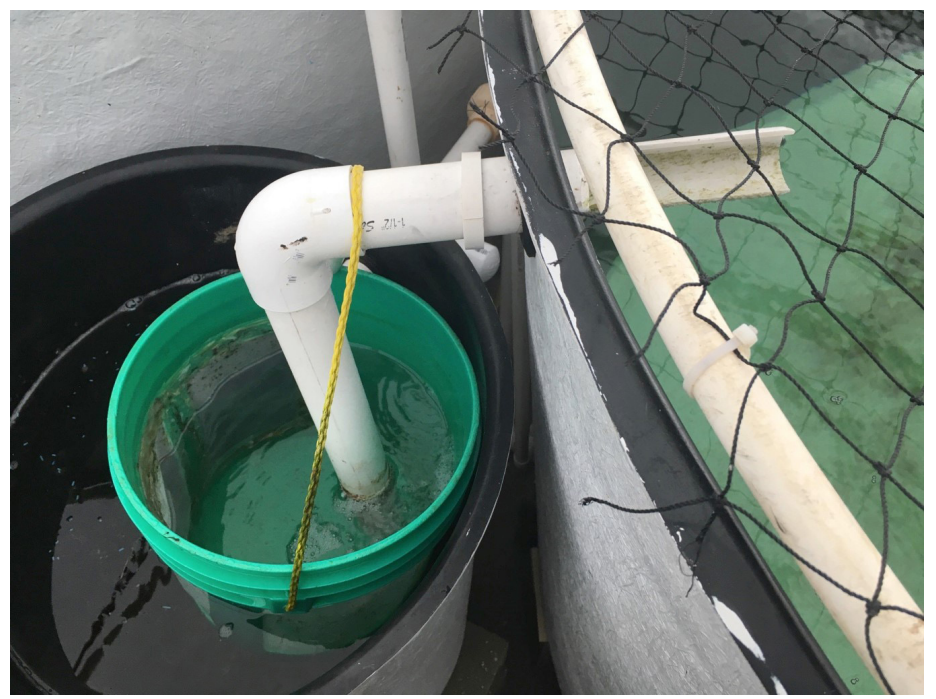

Figure 1. Overflow pre-filter egg collector with water overflowing from the skimmer pipe within the tank directly into screen mesh lined egg collector. Note the skimmer pipe in the tank is cut in half to collect eggs as they float towards the collector.

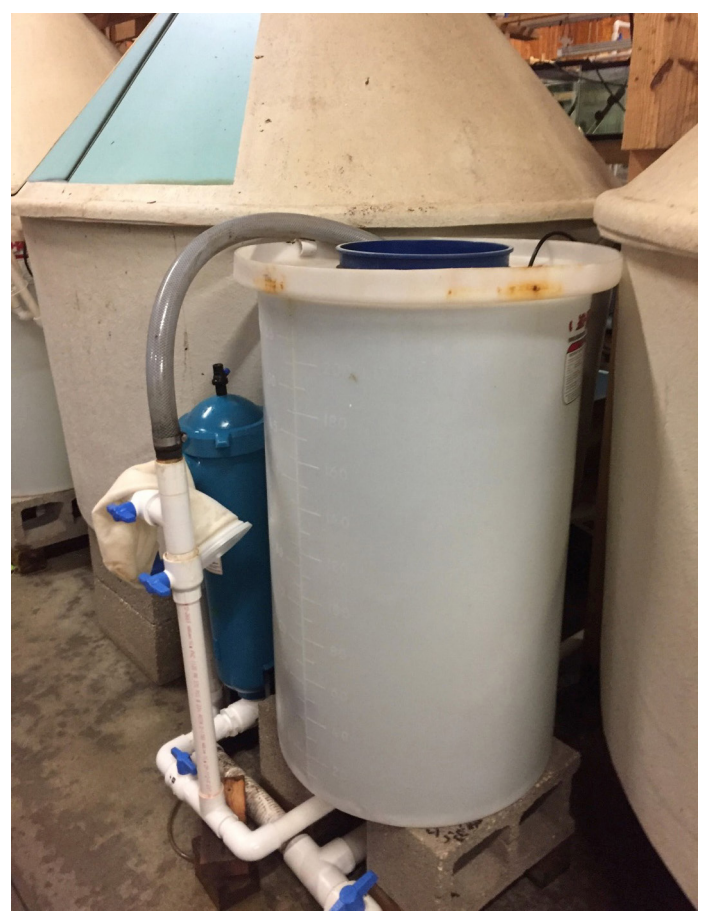

Figure 2. Culture tank (left rear) and the sump/collection tank (front center) containing an upwelling pre-filter egg collector.

Aquaculture systems can have overflows that lead directly to a sump. A simple way to test if spawning is occurring is to place a fine mesh net between the overflow and the sump where all, or most, of the water flows. It may also be possible to increase the water level in the sump so that the bottom of the net and the captured eggs are underwater, reducing potential damage. Ideally, a more efficient and less damaging method of capturing eggs should be used. However, this is a quick and simple method to determine 
whether a tank contains spawning fish, and some of the eggs collected may be viable.

Upwelling egg collectors (Figures 2 and 5) are typically designed to collect eggs from both the surface and bottom drains. By collecting both floating and sinking pelagic eggs, you are better able to understand the spawning efficiency of your broodstock. To remove the eggs from the collector, the bulkhead fitting should be capped with a PVC pipe or plug (Figure 6). Then the collector can be removed and the eggs poured and rinsed into a container.

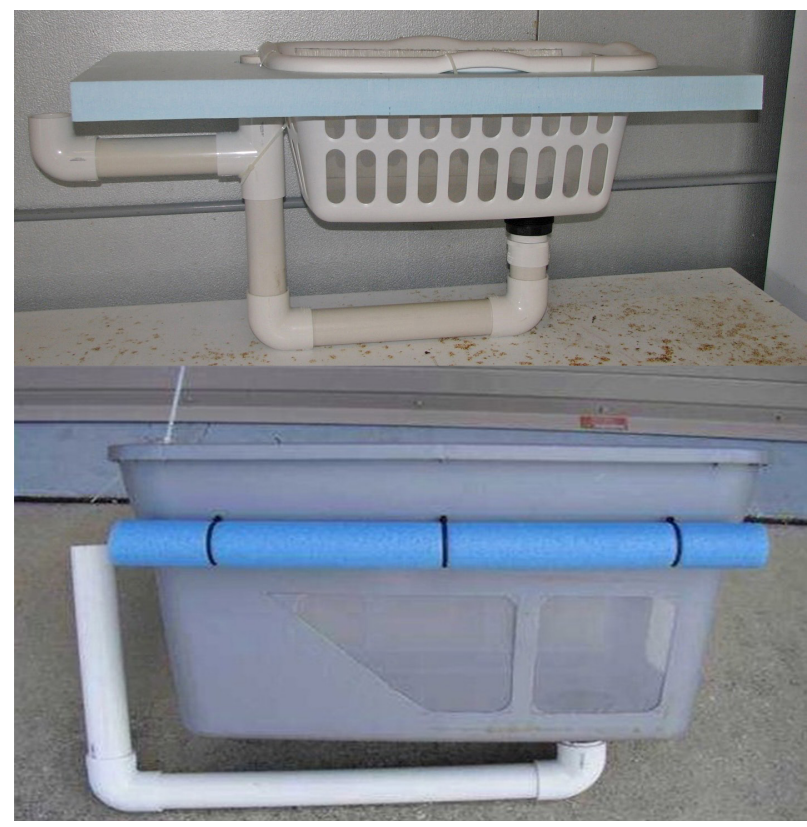

Figure 3. Photos of floating airlift egg collectors. (Top) The intake can be fine-tuned by placing a PVC pipe of appropriate lengths into the intake elbow to ensure it is $\sim 1 \mathrm{~cm}$ below the water surface when it skims eggs from the surface. (Bottom) Airlift egg collector constructed of a plastic bin and floatation provided by a swimming pool noodle.

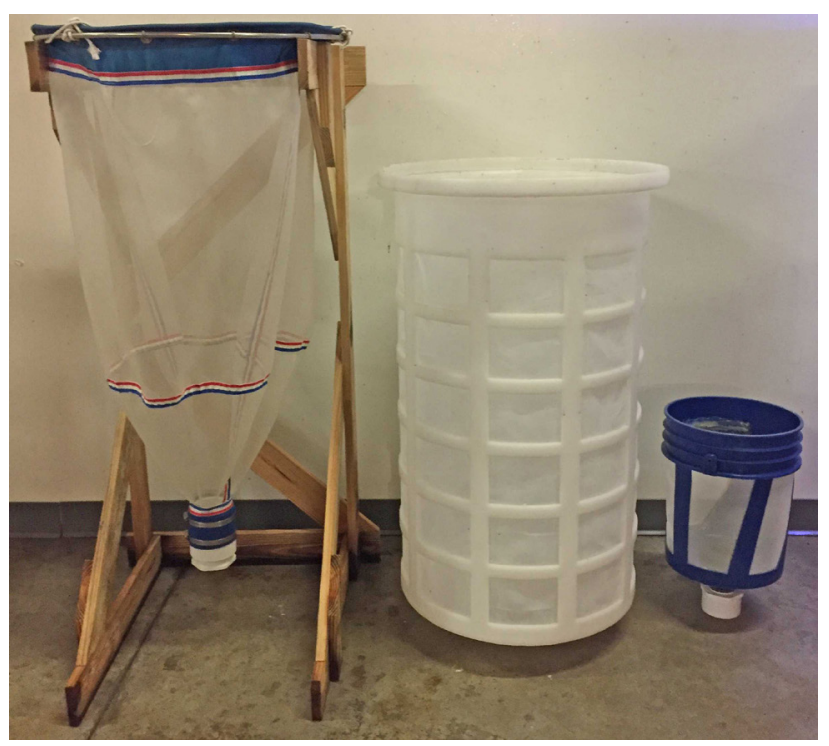

Figure 4. Alternatives to a bucket include a modified plankton tow net or a plastic barrel. These will provide greater mesh surface area and will accommodate larger spawns than a bucket.

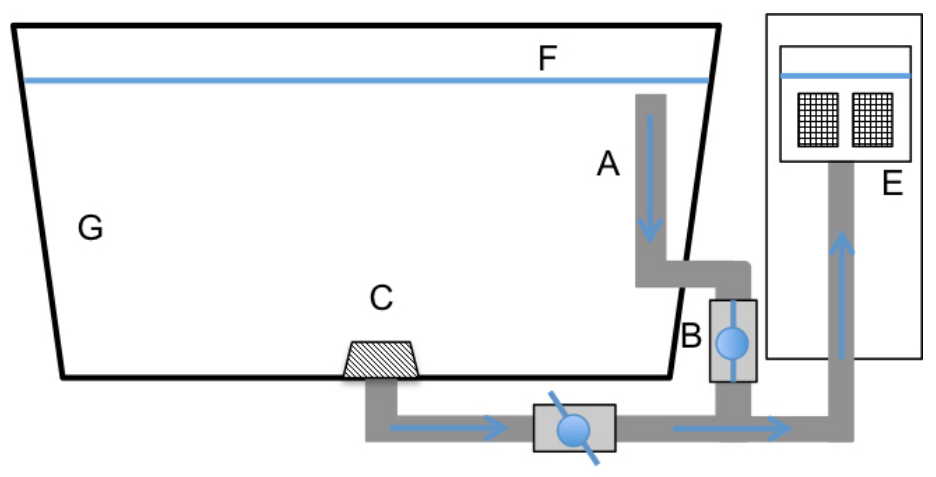

D

Figure 5. An upwelling pre-filter egg collector and culture tank holding the broodstock. A) skimmer pipe B) skimmer pipe valve C) bottom drain $\mathrm{D}$ ) bottom drain valve E) egg collector basket with $500 \mu \mathrm{m}$ nylon mesh screen F) water level G) culture tank containing broodstock. Arrows indicate direction of water flow.

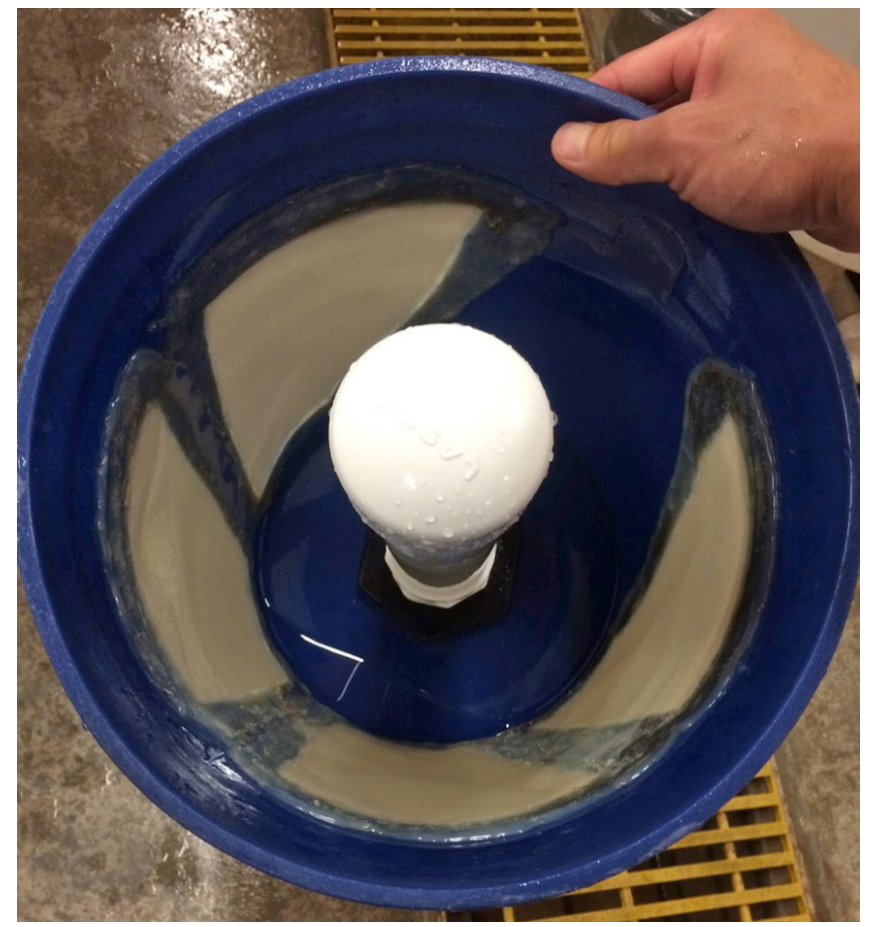

Figure 6. Upwelling pre-filter egg collector with a standpipe and cap used when removing the bucket for cleaning and egg collection. The standpipe prevents contents from exiting the bucket and entering the water flowing into the filtration system.

\section{PRE-FILTER EGG COLLECTOR- CONSTRUCTION}

Pre-filter egg collectors can be constructed from an appropriately sized plastic container, a nylon mesh screen, and a bulkhead fitting. A sturdy-walled plastic container, tote, bucket, or barrel can be used to construct a collector. The size of the collector depends on the size and water flow of the system as well as the quantity of eggs targeted for collection. For example, the plastic 5-gallon buckets pictured in Figures 1 and 2 sufficiently collect eggs from 500-700 gallon broodstock tanks housing 10-15 brood fish. Remove the walls of the collection containers with a cutting 
tool, such as a jigsaw or razor blade (Figure 7), leaving just enough vertical and horizontal sections of wall to provide attachment area and support for the nylon mesh screen. Smooth all rough edges around the cut areas by scraping and sanding to reduce abrasion of the screen. Notice that one wall of the bucket remains intact to provide an area to concentrate, rinse, and pour out eggs. This prevents eggs from being stranded on the screen while you are removing them from the collector.

If you are constructing an upwelling egg collector, attach a bulkhead to the bottom of the bucket (Figure 7). Then, attach a male PVC slip adapter to the bulkhead on the outside of the bucket, which can be slipped onto the standpipe within the sump. Use a hole-saw, a drill, or a jigsaw to remove the proper sized hole for the bulkhead. Scrape and sand smooth all the rough edges around the hole.

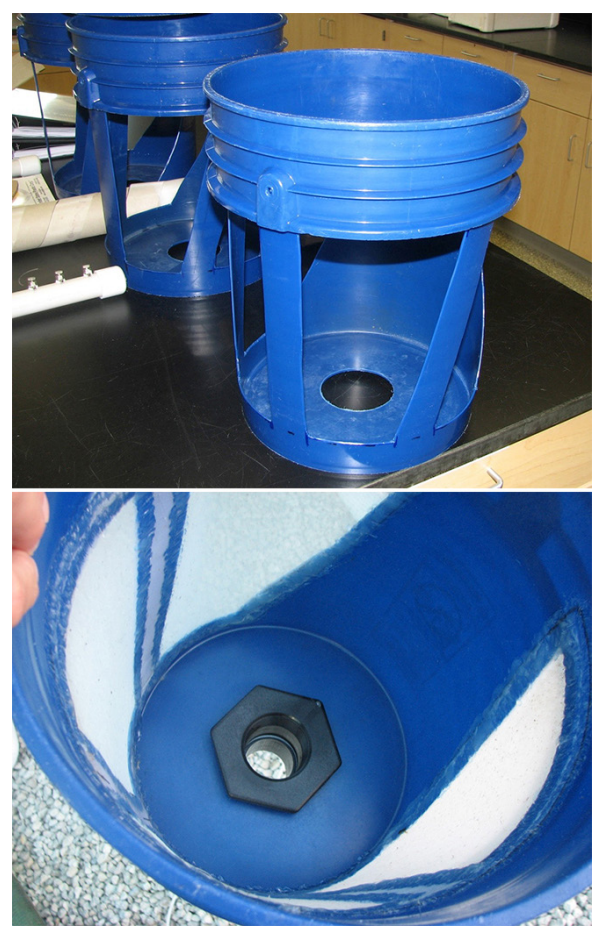

Figure 7. (Top) Five gallon bucket with openings removed and vertical areas for nylon mesh screen attachment. (Bottom) Egg collector bucket showing attached nylon mesh screen and bulkhead for attachment to discharge pipe within the sump.

For all egg collectors, choose a nylon screen mesh diameter that is smaller than the egg diameter of the target species. The mesh size should be $40 \%-60 \%$ of the diameter of the eggs to prevent them from getting damaged in the mesh. For example, a $500 \mu \mathrm{m}$ (micron) mesh would be used for an egg with an 800-1000 $\mu \mathrm{m}$ diameter. Attach the nylon mesh screen to the inside of the bucket as smoothly as possible with hot glue to prevent damage or entrapment of eggs. Apply the hot glue in multiple thin layers until the screen is sealed against the container walls. When gluing, ensure there are no remaining areas for eggs to escape or become entrapped along the edges where the container walls and mesh meet (Figures 6 and 7). It is possible to use non-toxic silicone to attach the screen, but it dries slowly compared to hot glue, so use special care to prevent the screen from moving while the silicone dries.

\section{Floating Airlift Egg Collector}

In contrast to pre-filter egg collectors, which are built into a recirculating aquaculture system, floating airlift egg collectors are a separate component usually placed into a tank or sump of a recirculating aquaculture system. Airlift collectors use rising air bubbles to create water movement through a PVC pipe that draws water (and any eggs in it) up and over a standpipe and into a mesh-lined collector (Figures 3 and 8). A skimmer pipe outside of the egg collector draws water from just below the water's surface $(\sim 1 \mathrm{~cm})$ to concentrate floating eggs inside the collector. Therefore, the rate of aeration can be used to regulate suction and water flow into the egg collector.

Floating airlift egg collectors can be constructed from any adequately sized plastic container, nylon mesh screen, PVC pipe and fittings, and a bulkhead. The size and shape of the egg collector can vary depending on the tank you are targeting. You will also need an air source and airline tubing to operate an airlift-driven egg collector. The diameter of the PVC pipe, the diameter of the air bubbles, and the amount of aeration will determine the flow rate of the water and efficiency of the collector. We recommend a PVC pipe diameter between $3 / 4$ inch and 2 inches. Any smaller and you will have trouble fitting an appropriately sized air stone into the internal standpipe. Any larger and the air flow will have to be increased to efficiently function, which increases turbulence and can damage collected eggs. You may need to test different fittings on the intake pipe that can increase collection surface area without causing additional damage. You will need a small air stone and airline tubing. Place these inside the internal standpipe to provide suction and water flow into the collector (Figure 8). For floating airlift egg collectors without a nearby air source or electrical outlet, a battery-operated air pump can be attached to the top of the basket with a cable tie.

Remove eggs from the collector by skimming or dipping them out with a pitcher, beaker, or cup. You can also simply lift the basket out of the tank and allow most of the water to drain out of it, carefully tip the basket to concentrate the remaining water and eggs in one corner, then gently pour and rinse the eggs into a pitcher, beaker, or cup. 


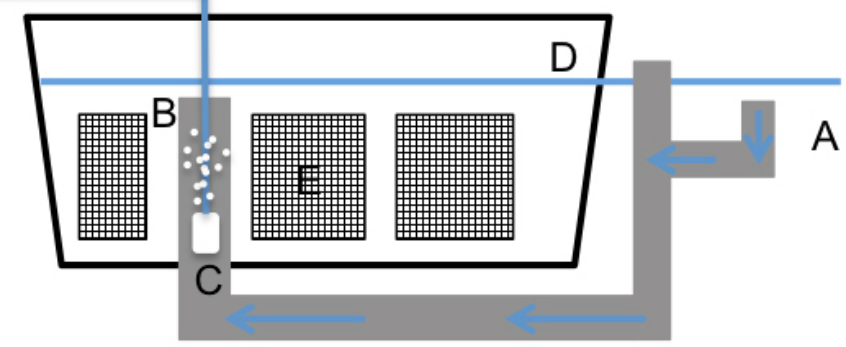

Figure 8. Floating airlift egg collector. A) skimmer intake B) standpipe with inflow of water C) air stone D) water level E) $500 \mu \mathrm{m}$ nylon mesh screen F) air release pipe. Arrows indicate direction of water flow.

\section{FLOATING AIRLIFT EGG COLLECTOR- CONSTRUCTION}

The construction of a floating airlift egg collector is similar to that of the pre-filter egg collector described in the Prefilter Egg Collector - Construction section. If it fits your tank, a plastic laundry basket makes a good floating airlift egg collector. These are usually manufactured with molded holes that provide the necessary surface area to secure the nylon mesh screen. They also have a strong framework to support the screen while in operation. If you use another plastic container, follow the directions in the Pre-filter Egg Collector - Construction section for removing container walls and leaving areas for support and attachment of the screen. As for the upwelling pre-filter egg collector, you will need to install a bulkhead in the bottom of the collection device to connect pipework inside and outside of the collector.

A foam pool noodle or a section of foam insulation $(\sim 2$ inches thick) can be custom cut to provide flotation (Figure 3). Use plastic zip ties to attach the foam to the egg collector. Position the floating egg collector in the water so that the intake pipe is about a centimeter below the surface, ensuring that the airlift suction will collect floating eggs. The height of the intake pipe can also be adjusted by placing a PVC pipe of appropriate length into the intake elbow (Figure 8). Attach an open PVC pipe to the intake pipe and extend it above the water's surface to provide an air escape. If air accumulates in the intake pipe, it will rise out of the water and stop collecting eggs.

The air flow can be adjusted to achieve the desired water flow for collecting eggs. If the air flow is too low, then the water flow will not be high enough to create suction and pull the eggs into the collector. If the air flow is too high, then the turbulence inside the egg collector may damage the eggs. Typically, using smaller air bubbles will increase water flow and decrease turbulence. Additionally, having a high air flow does not necessarily create a high water flow. Perform a few tests to ascertain the efficacy of your collector, but usually a small, gentle vortex created at the intake will collect floating eggs without causing damage.

\section{Maintenance}

Egg collectors get dirty quickly because they collect more than eggs. Any debris large enough to be retained within the collector will accumulate. Depending on system design, the egg collector may need to be removed and rinsed with freshwater several times a day. This will prevent the nylon mesh screen from becoming clogged. Cleaning and maintenance will ensure that the screen remains effective. Rinse and bleach the egg collector regularly depending on the amount of fouling that occurs. Some collectors may need to be bleached daily, while others can be done weekly. Over time, bleaching will damage the nylon mesh screen material and limit the life of the collector, so develop a good protocol that reduces fouling when appropriate. If the collector is not too dirty, a freshwater rinse and letting it sit to dry for $\sim 12$ hours should be sufficient to prevent the accumulation of fouling without damaging the collector. Your collector will last longer if you remember to remove it from the system and allow it to air dry whenever you are not using it to collect eggs. It's a good idea to make back-up collectors. If you have at least two collectors for each tank, then while one is in use, the other can be drying, reducing the need for bleach and extending the life of both collectors.

\section{Efficiency}

Egg collectors are generally very efficient at removing floating eggs. Their efficiency depends on the flow patterns, flow rate, and size of the broodstock tank. Directional flow of air and lengthwise, half-cut PVC pipe on the surface skimming intakes can move floating eggs towards the egg collectors. Timing is also of critical importance when considering egg collector efficiency. Some species spawn at a particular time of day. For example, many marine species spawn at dusk, so egg collecting can begin at that time and go through the night. Become familiar with the spawning patterns of your particular species of fish. Each system can vary as well; a longer collection time will be required for an airlift collector in a 10,000-gallon aquarium exhibit than it would for an overflow collector in a 500-gallon tank. Multiple factors must be considered to determine how to maximize the collector's efficiency.

\section{Conclusion}

To develop successful captive breeding programs for new species of marine fish, we must determine the unique needs of these species and evaluate whether they will be good candidates for aquaculture. For many marine fish species, 
egg production and larval development are major limitations in defining aquaculture protocols, particularly for pelagic spawning fish species. Access to numerous, viable pelagic eggs is necessary in the development of aquaculture production techniques for many pelagic spawning marine fish species. Many factors have to be considered when collecting pelagic eggs out of fish tanks and aquariums. The multitude of tank sizes, water flow rates, and staffing are just a few factors to consider when collecting eggs. Variations of two inexpensive, easy-to-use egg collector designs were presented and have been proven to work within many culture systems from 50-gallon tanks to huge public aquaria containing thousands of fish. Use these designs or modify as necessary to fit into specific tank filtration systems.

\section{Recommended Readings}

Cassiano, E. J., M. A. DiMaggio, C. L. Ohs, and J. Marcellus. 2015. FA188. Using airlifts to collect and concentrate copepod nauplii. Gainesville: University of Florida Institute of Food and Agricultural Sciences. http://edis.ifas.ufl.edu/fa188

Wittenrich, M. L. 2007. The complete illustrated breeders guide to marine aquarium fishes. TFH Publications, Neptune, NJ. 